\title{
Image segmentation for vegetation types extraction using WorldView-2: a case study in parts of Dieng Plateau, Central Java
}

Siti Martha Uly Br Sinaga, Muhammad Kamal

Siti Martha Uly Br Sinaga, Muhammad Kamal, "Image segmentation for vegetation types extraction using WorldView-2: a case study in parts of Dieng Plateau, Central Java," Proc. SPIE 11311, Sixth Geoinformation Science Symposium, 113110R (21 November 2019); doi: 10.1117/12.2543480

EDIE Event: Sixth Geoinformation Science Symposium, 2019, Yogyakarta, Indonesia 


\title{
Image Segmentation for Vegetation Types Extraction using WorldView-2 (A Case Study in Parts of Dieng Plateau, Central Java)
}

\author{
Siti Martha Uly Br Sinaga ${ }^{* a}$ and Muhammad Kamal ${ }^{\mathrm{a}}$ \\ a'Department of Geographic Information Science, Faculty of Geography, \\ Universitas Gadjah Mada, Yogyakarta, Indonesia 55281
}

\begin{abstract}
Image segmentation is the most important stage on Geographic Object Based Image Analysis (GEOBIA). The result of segmentation affects the final accuracy of classification. One of the applications of image segmentation operations is to delineate vegetation objects. Further analysis of vegetation could be used for inventory of natural resources, agricultural, land cover, land use, etc. However, applying image segmentation for separating vegetation types is challenging due to their irregular shapes and various patterns and colors. This study aims to determine the optimum parameters of image segmentation for delineating vegetation types using a pan-sharpened WorldView-2 image $(0.5 \mathrm{~m}$ pixel size $)$ which was acquired on August 2018. Combinations of scale parameter and composition of homogeneity criterion (shape and compactness) were systematically simulated to obtain the best segmentation parameters. The result of segmentation was assessed quantitatively based on visually interpreted image map as a reference. This study found that application of shape and compactness simultaneously for vegetation extraction would produce rough segmentation result. The optimum parameters for segmenting vegetation types using WorldView-2 were using scale parameter of 5, shape of 0 and compactness of 0.5 .
\end{abstract}

Keywords: segmentation, vegetation, WorldView-2, scale, shape, compactness

\section{INTRODUCTION}

Geographic Object Based Image Analysis (GEOBIA) is a sub-discipline of GIScience devoted to developing automated methods to partition remote sensing imagery into meaningful image-objects and assessing their characteristics through scale [1]. The main purpose of GEOBIA in the context of remote sensing application is to provide adequate and automated methods for the analysis of Very High Spatial Resolution (VHSR) imagery by describing the image reality using spectral, textural, spatial and topological characteristics. GEOBIA offers a methodological framework for machine-based interpretation of complex classes, defined by spectral, spatial and structural as well as hierarchical properties [2]. These methods are finding increasing popularity particularly when applied to high resolution satellite imagery [3]. Remote sensing analyses incorporating GEOBIA have become increasingly entrenched in the scientific literature [4]. The advantages of GEOBIA compared to the visual and pixel-based classification methods are the ability to incorporate multiple scales, better mimics human perception of objects, and able to integrates attributes important to landscape analysis (tone, shape, size, texture, context) [5].

GEOBIA has two main steps in creating objects. First step is segmentation, where pixels are merged to object clusters, possibly in multi-level object hierarchy, which then will be analysed and classified in the second step [6]. Segmentation is the underlying concept for creating object from pixels. The segmentation process involves dividing the image into regions or objects that have common properties [7]. Image segmentation decreases the level of detail in a scene, reduces images complexity, and makes image content more graspable [8]. Segmentation process has the greatest influence on the accuracy of the obtainable results, it is dependent on the segmentation algorithm, parameters and the appropriate observation scale. While classification is the final process of GEOBIA after obtained the optimum segmentation. Classification will produce the map which represent real world objects through meaningful image segments. 
The application of GEOBIA in various objects for spatial analysis has been widely used, such as land use/land cover mapping, building analysis, geologic study, urban study, vegetation identification, etc. This study would focus on recognizing the types of vegetation, specifically in highland area. Vegetation is one of the objects which can be extracted on high resolution imagery through GEOBIA. Classifying and mapping vegetation is an important technical task for managing natural resources as vegetation provides a base for all living beings and plays an essential role in affecting global climate change, such as influencing terrestrial $\mathrm{CO}_{2}[9]$. Further analysis of vegetation could be used for inventory of natural resources, agricultural, land cover/land use, etc. Therefore, an effective method for vegetation extraction would greatly help the process. The novelty of this study is the application of GEOBIA that can automatically separate different types of vegetation by considering various types of object characteristics on the image. Simulations of parameter combination would also be carried out to produce the optimum segmentation. The result of this study can be used for consideration in future research of vegetation mapping and analysis.

The aim of this study is to determine the optimum parameters of image segmentation for delineating vegetation types using a pan-sharpened WorldView-2 image ( $0.5 \mathrm{~m}$ pixel size) using GEOBIA. This study would only focus on segmentation process, while the classification will be discussed in other paper. The approach was applied to extract the types of vegetation in highland area, by involved Multiresolution Segmentation and assessed the segments accuracy quantitatively in order to know the quality of segmentation.

\section{METHODS}

\subsection{Study Area and Dataset}

This study was conducted in parts of Dieng Plateau, Central Java, Indonesia. The extent of the study area is between 377798 to 378410 meters East and between 9201400 to 9202200 meters North (based on UTM coordinate system zone 49M). The study area covers 42 ha which consist of agricultural land, forest land, and scrub area. The selection of this area because of the various vegetation types which making it possible to applied various object features on the GEOBIA process. The study area is shown in Figure 1.

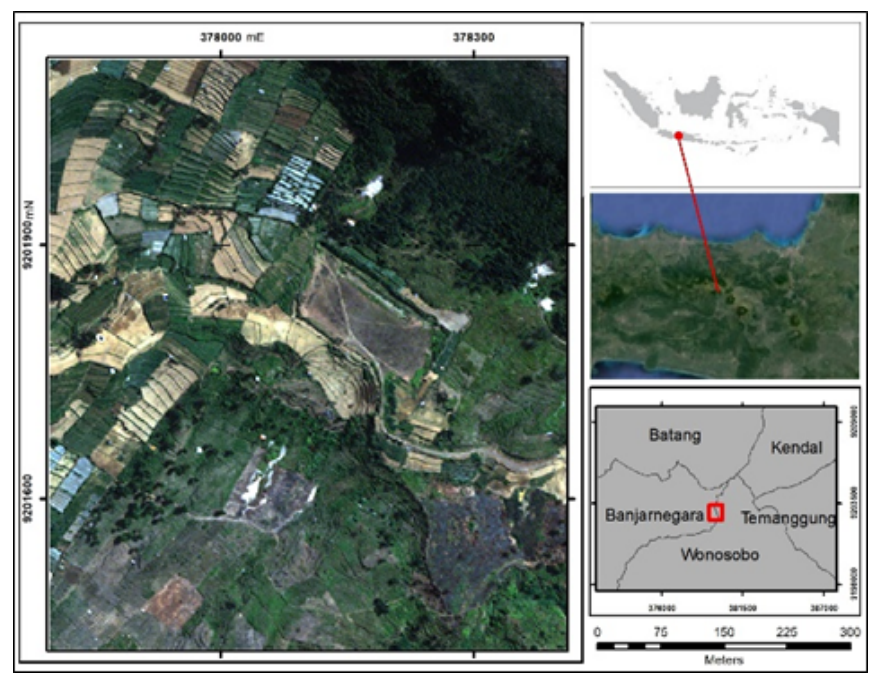

Figure 1. The map of study area

A WorldView-2 image acquired on August 6th, 2018 was used in this study. The image obtained from DigitalGlobe (www.digitalglobe.com) and available at "Standard2A" correction level which means had been corrected on geometric with brightness value digital number. Therefore, standard radiometric correction to surface reflectance value was needed. Image fusion using Gram-Schmidt pan-sharpening method also performed in this study to maximize the visual image quality from 1.86 meter to 0.5 meters pixel size. Image fusion only applied to $450-800 \mathrm{~nm}$ (Panchromatic) wavelength on multispectral bands, consist of blue $(450-510 \mathrm{~nm})$, green $(510-580 \mathrm{~nm})$, yellow $(585-625 \mathrm{~nm})$, red $(630-690 \mathrm{~nm})$, rededge $(705-745 \mathrm{~nm})$ and near-infrared $1(770-895 \mathrm{~nm})$. 


\subsection{Reference Data of Vegetation Types Polygons}

Reference data is needed to assess the accuracy of segmentation result. In this case, the reference data was obtained from visual interpretation of the WorldView-2 pan-sharpened image. Vegetation objects was delineated by on-screen digitization technique with considered the minimum mappable unit (MMU) rules, where the smallest object could be recognized from image is $2 \times 2 \mathrm{~mm}$ [10]. The targeted map output was 1:5,000, therefore the MMU in this study was 10 $\mathrm{x} 10 \mathrm{~m}$, the one of example of $10 \times 10 \mathrm{~m}$ objects in the field is part of agricultural field. The visual interpretation process was carried out up to 1:1,250 zooming scale level.

\subsection{Multiresolution Segmentation}

Multiresolution Segmentation was applied by combination of three parameters of the multiresolution segmentation algorithm that implemented in eCognition Developer 9.01 (www.ecognition.com). This algorithm was developed by Baatz and Schäpe [11] with the aim of reducing heterogeneity of images by creating object segments with accurate results even though carried out at various scales and data types. The selection of this algorithm due to the consideration of this study purpose which focused to find out the relevant parameters in determine the optimum parameters for delineating vegetation types. The segmentation algorithm is based on a region growing technique which places seed pixels over an entire image and groups surrounding pixels to the local seeds, if they meet specific criteria [11]. The aim of this algorithm is to reduce heterogeneity of image by creating objects segments with accurate results even though carried out at various scales and data types.

Multiresolution segmentation has two main components: (1) decision heuristics to regulate the image objects that will merge at each step and (2) definition of a homogeneity of image objects to calculate the degree of fitting for a pair of image objects [11]. This algorithm produces object segments based on scale parameter, color, shape, smoothness and compactness. Scale parameter is an abstract term that determines the maximum allowed heterogeneity for the resulting image objects. By modifying the value, the size of image objects can be varied. The value of the Shape field modifies the relationship between shape and color criteria. By modifying the shape, it means define the color criteria (color $=1$ shape). In effect, by decreasing the value assigned to the Shape field, means define to which percentage the spectral values of the image layers will contribute to the entire homogeneity criterion. The compactness is used to optimize image objects regarding the compactness. These criteria should be used when different image objects which are rather compact but are separated from non-compact objects only by a relatively weak spectral contrast. By modifying the compactness, it would define the smoothness criteria [6].

\subsection{Segmentation Quality Metrics}

The quality of segmentation results is important to know. The low accuracy of a segmentation results affects the accuracy of the classification results [12]. This study would assess the accuracy from the most optimal segmentation that have performed. The optimal segmentation result would be overlaid with reference map which have produced before. The following table (Table 1) will describe the metrics and formula for accuracy assessment.

Table 1. Description of segmentation quality metrics

\begin{tabular}{|c|c|c|c|}
\hline Metrics & Formulas & Explanation & Source \\
\hline $\begin{array}{l}\text { Over-segmentation } \\
\text { (OSeg) }\end{array}$ & $O S e g=\frac{\operatorname{area~} X i \cap Y j}{\text { area } Y j}$ & \multirow{2}{*}{$\begin{array}{l}\text { The value between } 0-1 \text {. Closer to } 0 \text { indicates the } \\
\text { segmentation is well-performed, while value of } 0 \text { indicates } \\
\text { the object is perfectly segmented. }\end{array}$} & \multirow{3}{*}[13]{} \\
\hline $\begin{array}{l}\text { Under-segmentation } \\
\text { (USeg) }\end{array}$ & $U S e g=\frac{\operatorname{area~} X i \cap Y j}{\text { area } X i}$ & & \\
\hline $\begin{array}{l}\text { Root Mean Square } \\
\text { (D) }\end{array}$ & $D=\sqrt{\frac{O S e g^{2}+U S e g^{2}}{2}}$ & $\begin{array}{l}\text { Evaluates the errors found in the segmentation results in a } \\
\text { value of } 0 \text { to } 1 \text {. The smaller the value of } D \text {, the smaller the } \\
\text { segmentation error. }\end{array}$ & \\
\hline Area fit index (AFI) & $A F I=\frac{\operatorname{area} X i-Y j}{\text { area } X i}$ & $\begin{array}{l}\text { The value between } 0 \text { to } 1 \text {. Smaller AFI values or closer to } 0 \\
\text { indicates the segmentation is well-performed. }\end{array}$ & [14] \\
\hline Quality rate $(\mathrm{Qr})$ & $Q r=\frac{\text { area } X i \cap Y j}{\text { area } X i \cup Y j}$ & $\begin{array}{l}\text { The value between } 0-1 \text {. Closer to } 1 \text { indicates the better } \\
\text { segmentation created. If the value of Qr equal to } 1 \text {, then the } \\
\text { segmentation is perfect. }\end{array}$ & {$[15]$} \\
\hline
\end{tabular}




\section{RESULT AND DISCUSSION}

\subsection{Reference Data of Vegetation Types}

A reference data needed for assess the quality of segmentation. In this study, the reference data produced from visual interpretation of vegetation types using pan-sharpened WorldView-2 (0.5-pixel size). Delineation of different types of vegetation was carried out by on-screen digitization based on some key interpretations, such as colors, texture, shapes, sizes, patterns, shadows and associations. The types of vegetation that found in the study area consist of agricultural land, bare land, forest, shrub and tree. Shadows were included in classification because in some case, they covered vegetation objects and would be affected the result of segmentation. The reference data from visual interpretation are shown in Figure 2. This data would be over-laid with the segmentation result to know the accuracy of segmentation.

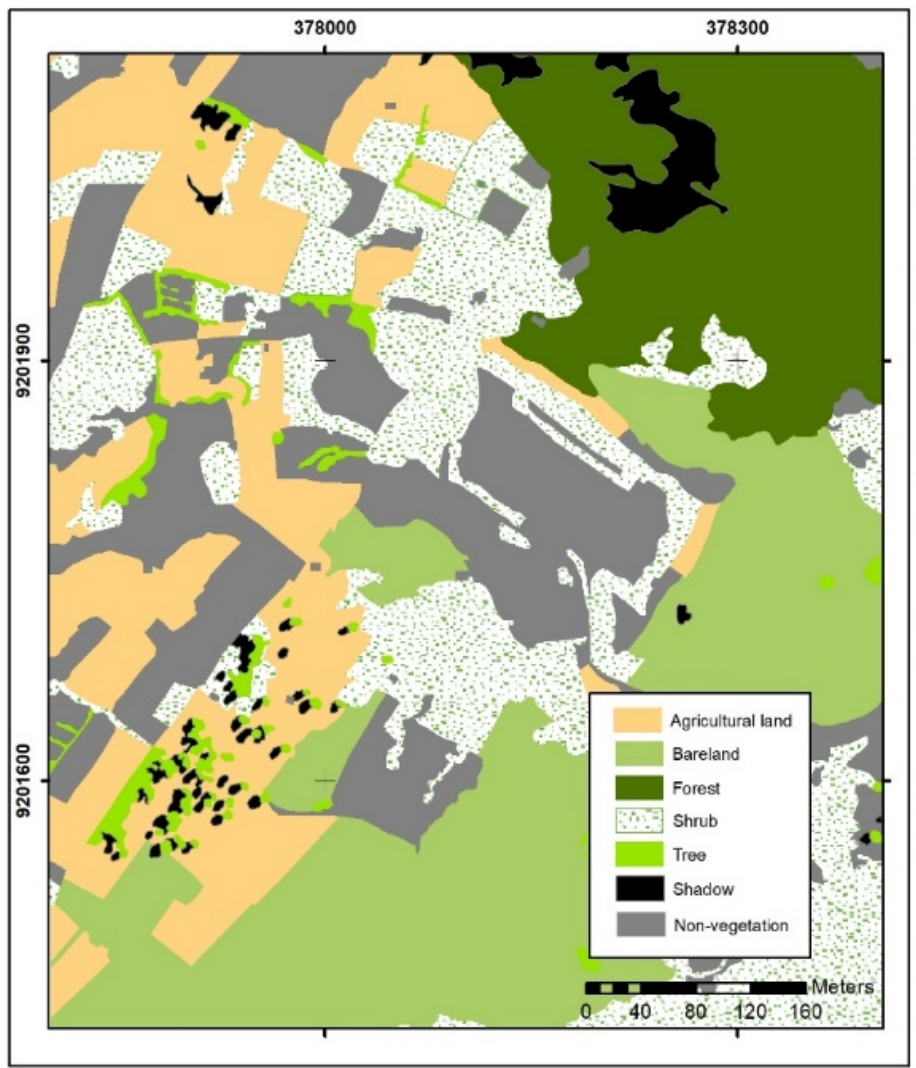

Figure 2. Reference data of vegetation types resulted from image visual interpretation.

\subsection{Multiresolution Segmentation}

Segmentation is the first step in GEOBIA which determine the object boundaries. This study focuses on recognizing the types of vegetation by applied multiresolution segmentation to WorldView-2 pan-sharpened image. Multiresolution segmentation was performed using scale parameter and composition of homogeneity criterion, consists of shape and compactness. The segments will be more detailed as the scale parameter decreases. Simulations by combining of various parameters was carried out to get the optimal segmentation (i.e. able to properly separate different types of vegetation). Based on the image, the types of vegetation can be distinguished from the textures and shapes, therefore the first simulation applied the same weight of shape and compactness criterion. The results of segmentation created from different scale parameters can be seen in Figure 3. 

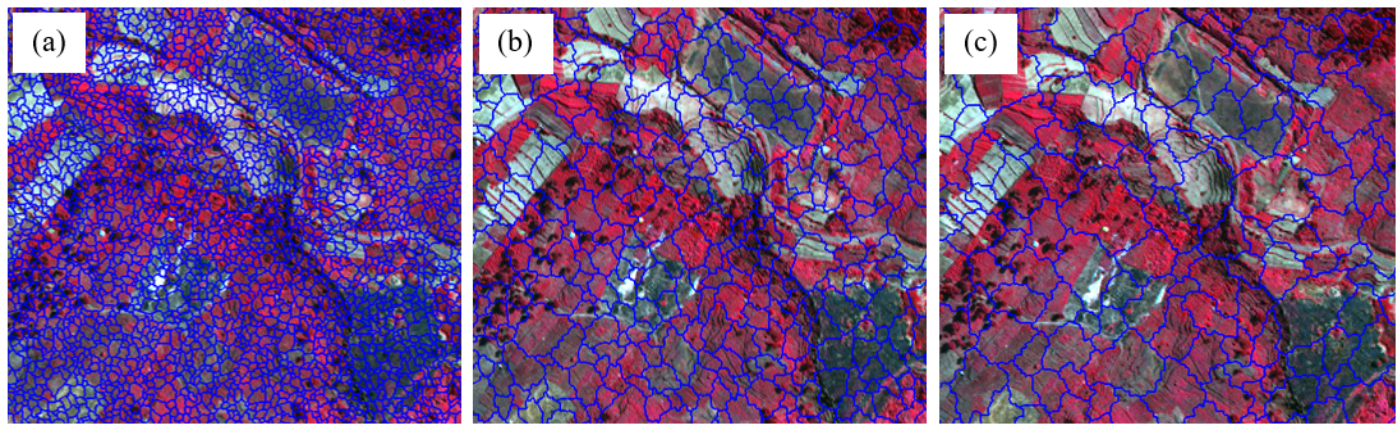

Figure 3. Segmentation created from different scale parameters (with shape:compactness ratio of 0.5:0.5);

(a) SP 10, (b) SP 30, and (c) SP 50.

The results of segmentation in Figure 3 show poor segmentation with rounded segments. Segmentation using the shape and compactness criterion coincidely to the image were not appropriate. The object boundaries were too rough and not able to separated objects properly.

The other simulations used only shape criterion with various weight (with compactness $=0$ ). The higher weight given to the shape criterion, the more detailed segments would be produced. This simulation produced better separated segments for the different objects, particularly on regular shapes object as agricultural land (Figure 4). However, this segmentations were not able to properly separate trees and irregular vegetation objects.
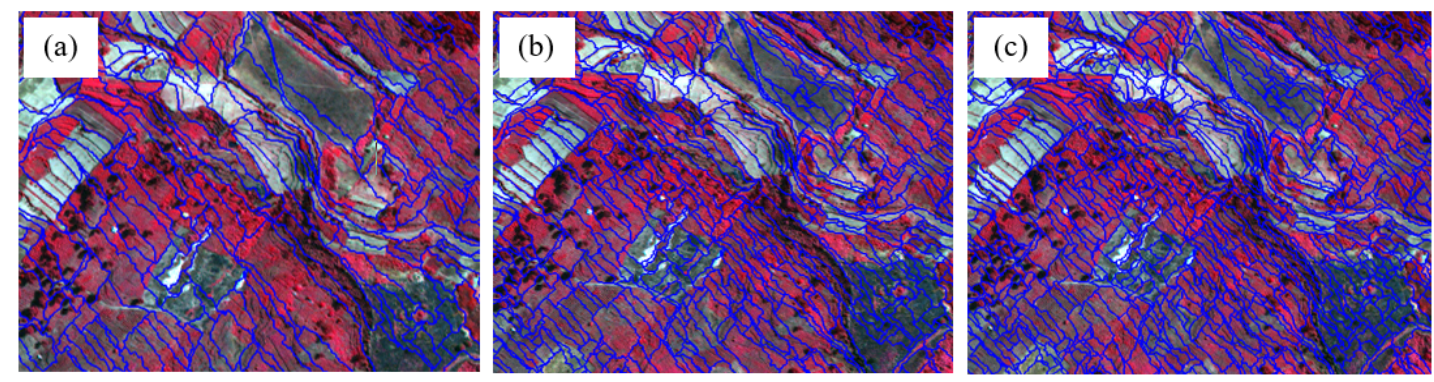

Figure 4. Segmentation created from different weights of compactness (with shape $=0$ and scale parameter $=20$ );

$$
\text { (a) } 0.2 \text {, (b) } 0.5 \text {, and (c) } 0.8 \text {. }
$$

Simulations were also applied using only compactness criterion (with shape $=0$ ). The results show the best segmentation that could separated the different types of vegetation according to the actual border. The weight that given to the compactness criterion was 0.5 and there was no significant segments change when applied different weight. Therefore trials and errors to obtain the most optimal segments were carried out by adjust the scale parameters. Figure 5 shows the segments that increasingly detailed as the weight of scale parameter decreases.
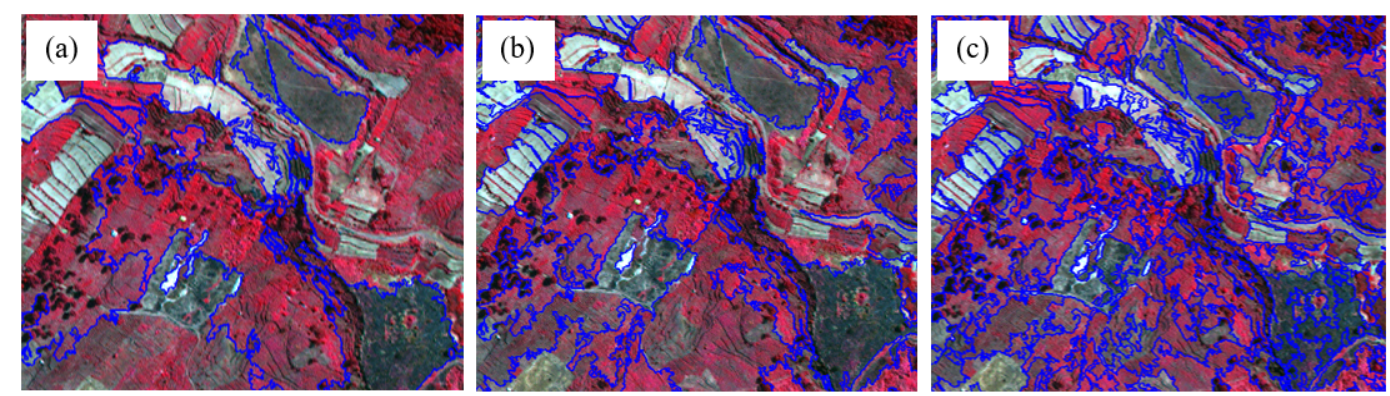

Figure 5. Segmentation created from different $S P$ (with shape:compactness =0:0.5); (a) 20, (b) 10, and (c) 5 . 
Based on Figure 5, by applied the compactness weight $=0.5$, means the smoothness criterion weight $=0.5$. In this case, the position of these criterions were balanced. Both compactness and smoothness were important in defining vegetation objects in the study area. Between three segmentations in Figure 5, the result of scale parameter of 5 (with shape:compactness $=0: 0.5$ ) are the most optimal segmentation. The segments could separated different types of vegetation, consist of agricultural land, bare land, forest, shrub and tree (Figure 6).
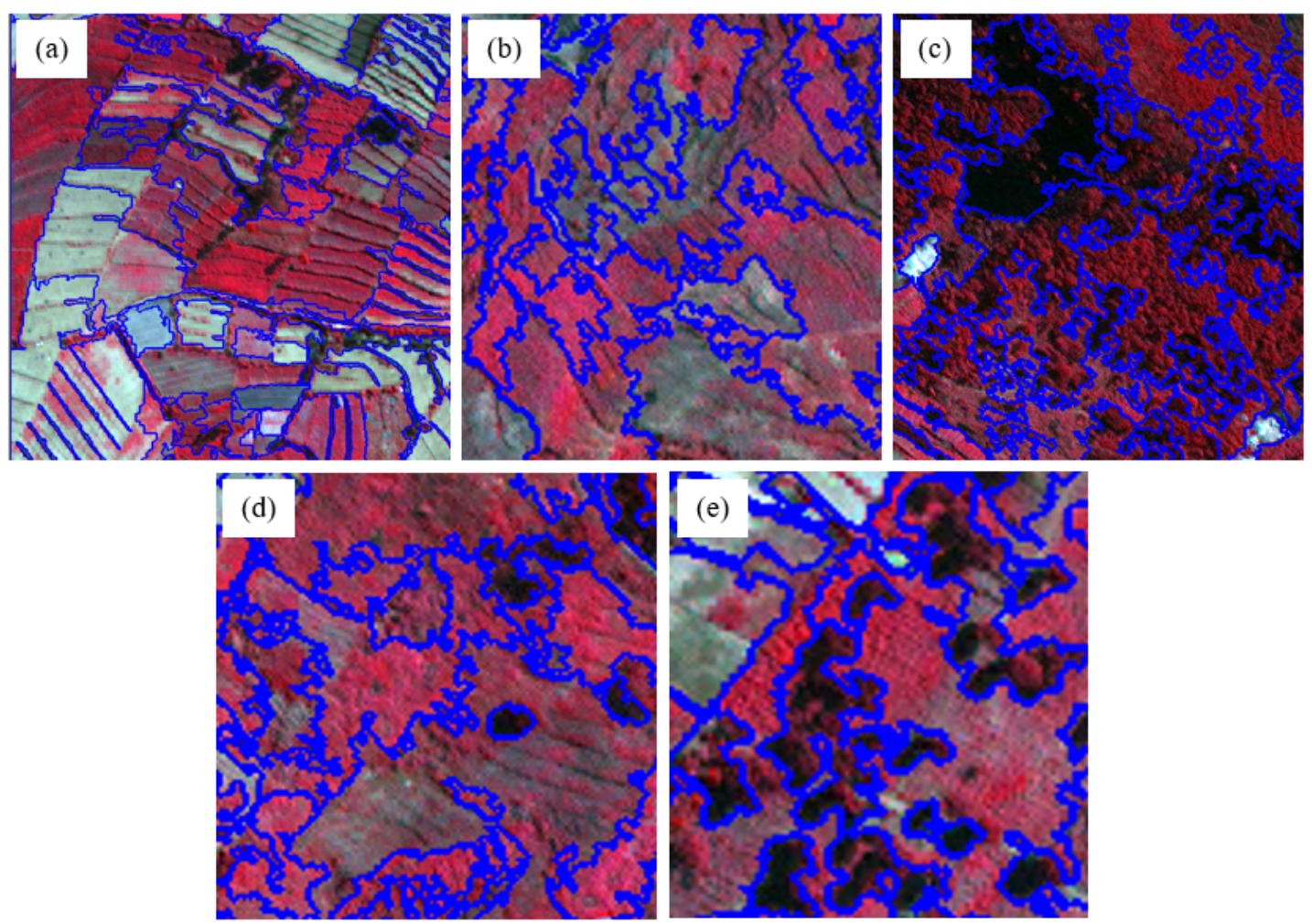

Figure 6. The segmentation of types vegetation: (a) agricultural land, (b) bare land, (c) forest, (d) shrub and (e) tree.

\subsection{Segmentation Quality Metrics}

The optimal segmentation with the scale parameter of 5 (with shape:compactness $=0: 0.5$ ) would be assesst in order to know the quality of segments. The detailed of accuracy can be seen in Table 2 .

Table 2. The quality of optimal segmentation result

\begin{tabular}{|c|c|c|c|c|c|}
\hline Selected Segmentation & OSeg & USeg & D & AFI & Qr \\
\hline $\begin{array}{c}\text { Scale parameter }=5 \\
\text { Shape }=0 ; \text { Compactness }=0.5\end{array}$ & 0.78 & 0.04 & 0.39 & 0.74 & 0.67 \\
\hline
\end{tabular}

Based on the calculation of segmentation quality, this study produced 0.67 of Quality rate (Qr) on segmentation result or around $67 \%$. The low accuracy in this case due to the considerable over-segmentation which also causes the high AFI value. However, the value of under-segmentation is very low which means almost all of vegetation types on reference data could be separated properly.

\section{CONCLUSION}

The optimum parameters of image segmentation for delineating types of vegetation obtained by trials and errors of several parameters in multiresolution segmentation. The segmentation is considered optimal when it is able to properly 
separate different types of vegetation. The result of segmentation could separated different types of vegetation, consist of agricultural land, bare land, forest, shrub and tree, by applied the scale parameter of 5 (with shape:compactness $=0: 0.5$ ). The accuracy produced 0.67 of Quality rate due to the considerable over-segmentation.

For future research, a larger study area and more variety of vegetation is needed to complement the GEOBIA studies literature for various conditions of vegetation. Application to higher resolution imagery as high-resolution aerial photography and tested various segmentation algorithms also needed to improve the accuracy.

\section{ACKNOWLEDGEMENT}

The authors would like to thank the Department of Geographic Information Science, Faculty of Geography, Universitas Gadjah Mada for supporting this research, RTA-2019 (Rekognisi Tugas Akhir) Grant from Universitas Gadjah Mada, contract number 3061/UN1/DITLIT/DIT-LIT/LT/2019 for providing funding for this study, and DigitalGlobe for providing free access to the WorldView-2 image data.

\section{REFERENCES}

[1] Castilla G. and Hay G. J., "Image objects and geographic objects," In Blaschke T., Lang S. and Hay G. J., [Object-based image analysis: Spatial concepts for knowledge-driven remote sensing applications], Berlin: Springer. (2008).

[2] Benz, U., Hofmann, P., Willhauck, G., Lingenfelder, I., and Heynen, M., "Multi-resolution, object-oriented fuzzy analysis of remote sensing data for GIS-ready information," ISPRS Journal of Photogramm Remote Sens. Papers 58(1), 239-258 (2004)

[3] Lennartz, S. P., and Congalton, R. G., "Classifying and mapping forest cover types using IKONOS imagery in the norteastern United States," in ASPRS Annual Conference Proceedings. (2004).

[4] Blaschke, T. "Object based image analysis for remote sensing," ISPRS Journal of Photogramm Remote Sens. Papers 65(1), 2-16 (2010).

[5] Morgan, J. L., Gergel, S. E. and Coops, N. C., "Aerial photography: a rapidly evolving tool for ecological management", BioScience. Papers 60(1), 47-59 (2010).

[6] Baatz, M., Benz, U., Dehghani, S., Heynen, M., Holtje, A., Hofmann, P., Lingenfelder, I., Mimler, M., Sohlbach, M., Weber, M. and Willhauck, G. [eCognition User's Guide 4], München: Definiens Imaging $\mathrm{GmbH}(2004)$.

[7] Navulur, K., [Multispectral Image Analysis Using the Object-Oriented paradigm], Boca Raton, Florida. (2007).

[8] Lang, S., "Object-based image analysis for remote sensing applications: Modeling reality - dealing with complexity," In Blaschke T., Lang S. and Hay G.J., [Object-based image analysis: Spatial concepts for knowledge-driven remote sensing applications], Berlin: Springer, 3-28 (2008).

[9] Xiao X. M., Zhang Q., Braswell B., "Modeling gross primary production of temperate deciduous broadleaf forest using satellite images and climate data," Remote Sens. Environment. Papers 91, 256-70 (2004).

[10] Lewinski S., and Bochenek, Z., "Rule-based Classification of SPOT Imagery Using Object-oriented Approach for Detailed Land Cover Mapping," Proc. The 28th EARSeL Symposium, Remote Sensing for a Changing Europe, (2008).

[11]Baatz, M. and Schäpe, A., "Multiresolution segmentation: An optimization approach for high quality multiscale image segmentation," Angewandte Geographische Informationsverarbeitung. Papers 12, 12-23 (2000).

[12] Liu, D. and Xia, F., "Assessing object-based classification: advantages and limitations," Remote Sens Lett. Papers 1(4), 187-194 (2010).

[13] Winter, S., "Location similarity of regions," ISPRS J Photogramm Remote Sens. Papers 55, 189-200 (2000).

[14] Lucieer, A. and Stein, A., "Existential uncertainty of spatial objects segmented from satellite sensor imagery," Geosci. Remote Sens., IEEE Trans. Papers 40, 2518-2521 (2002).

[15] Weidner, U., "Contribution to the assessment of segmentation quality for remote sensing applications," Int. Archives of Photogramm, Remote Sens. and Spatial Information Sciences. Papers 37(B7), 479-484 (2008). 